\title{
Siliziumkarbidhalbleiter mit breitem Bandabstand für Elektrofahrzeuge
}

Die Nachfrage der Verbraucher nach Elektrofahrzeugen steigt stetig - und damit auch die Nachfrage nach neuen Lösungen, um die Reichweite der Fahrzeuge zu maximieren und die Kosten zu senken. United Silicon Carbide hat Siliziumkarbidhalbleiter entwickelt, mit denen effizientere elektrische Antriebsstränge und Leistungsbauteile realisiert werden können. 


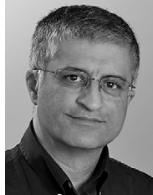

Anup Bhalla ist Vice President of Engineering bei United Silicon Carbide Inc. in Princeton (USA).

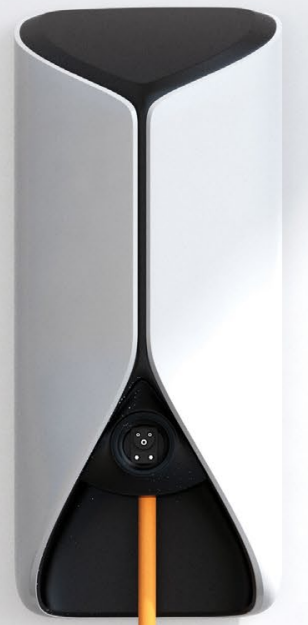

Elektroautos werden aufgrund der sinkenden Preise und steigenden Reichweiten immer beliebter. Im Jahr 2019 wurden weltweit mehr als 2,1 Millionen Elektroautos verkauft. Laut [1] waren somit im Jahr 2019 über 7,2 Millionen elektrische Pkw auf den Straßen unterwegs. Das weitere Wachstum hängt jedoch von einer Reihe von Faktoren ab, darunter dem Fortbestand der Kaufsubventionsprogramme in Schlüsselmärkten und die Auswirkungen von Covid-19 auf die Automobilproduktion. Eine weitere wichtige Herausforderung besteht im Ausbau der nationalen Ladeinfrastrukturen, die zur Unterstützung eines schnellen Ladevorgangs unterwegs erforderlich sind.

\section{HALBLEITER MIT BREITEM BANDABSTAND}

Batterie- und Motorenhersteller stoßen inzwischen an physikalische Leistungsgrenzen, die mit den etablierten Technologien, vor allem solchen, die Silizium verwenden, erreicht werden können. Bei Elektrofahrzeugen ist der wichtigste Bereich bezüglich Wirkungsgrad dort, wo die Gleichspannung der Batterie in dreiphasige Wechselspannung für die Motoren umgewandelt wird. Überall dort, wo Leistung zwischen DC- und AC-Seite umgewandelt wird, insbesondere bei Gleichspannungen im Bereich von $800 \mathrm{~V}$, wie sie in Elektrofahrzeugen benötigt werden, besteht ein erhebliches Verlustpotenzial. Eine Verbesserungsmöglichkeit, die die meisten OEMs von Elektrofahrzeugen hier in Betracht ziehen, besteht in der Umstellung von Siliziumhalbleitern auf Halbleiter mit breitem Bandabstand (Wide Bandgap, WBG), wie zum Beispiel Siliziumkarbid(SiC)-

Halbleiter.

Hinsichtlich der Leistungsumschaltung bieten SiC-Halbleiter bereits seit Längerem viele Vorteile. Aus diesem Grund wurden diese Halbleiter in Elektrofahrzeugen bisher vor allem für Anwendungen mit geringem Stromverbrauch eingesetzt, wie zum Beispiel Batterieladegeräte, Hilfs-DC/DC-Wandler und Halbleiterschutzschalter. Für den Antriebsstrang wurden SiC-Halbleiter unter anderem aufgrund des zu hohen Einschaltwiderstands und einer zu geringen Robustheit bisher nicht eingesetzt. Die aktuelle Generation von SiC-FETs von United Silicon Carbide ist nun auch für Hochleistungsanwendungen aufgrund des Aufbaus als gestapelte Kaskoden (Stacked Cascodes) geeignet [2].

\section{AUFBAU VON GESTAPELTEN KASKODEN}

Eine gestapelte Kaskode besteht aus der Reihenschaltung eines Hochspannungs-SiC-JFETs mit einem optimierten Niederspannungs-Si-Mosfet, BILD 1. Der Mosfet ist eingeschaltet, sobald

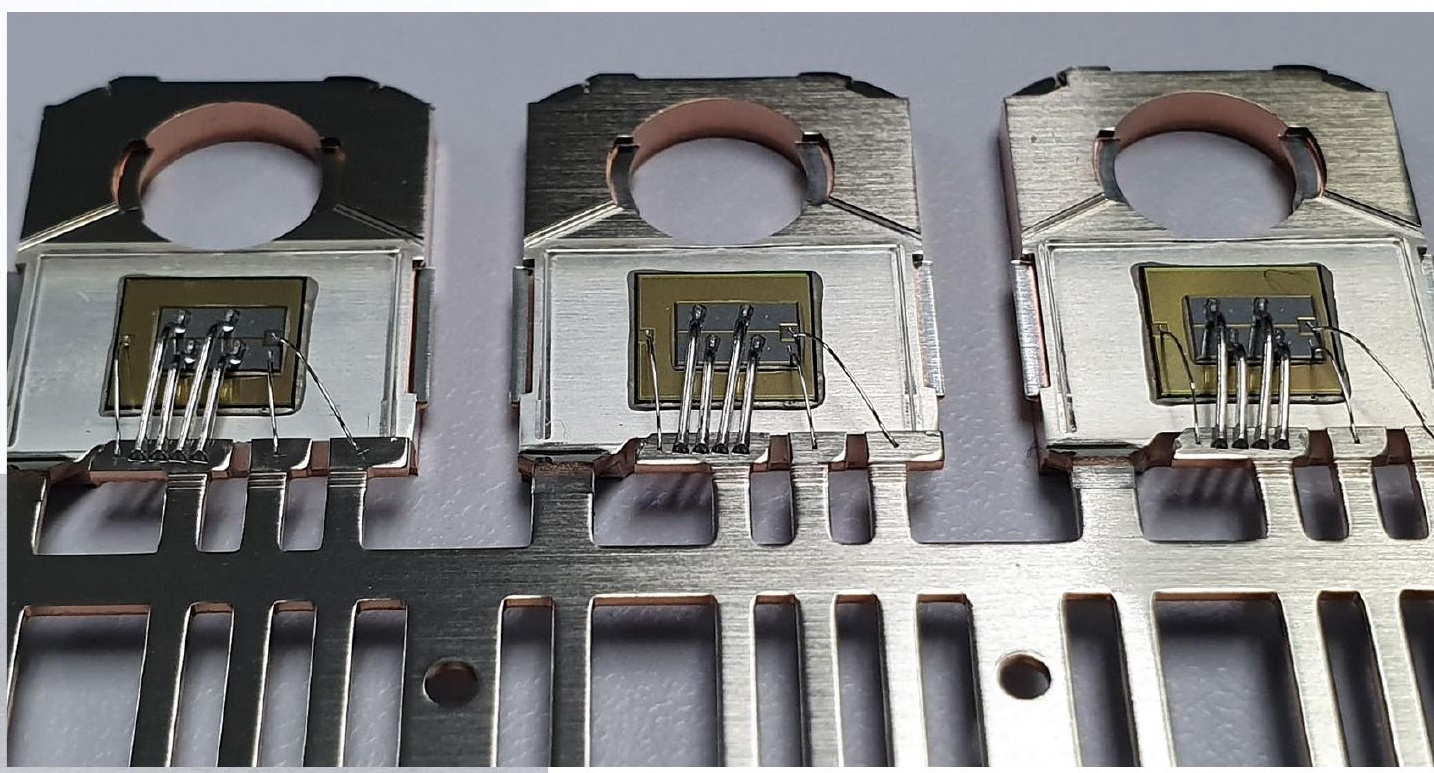

BILD 1 Aufbau einer gestapelten Kaskode - der Mosfet-Chip ist physikalisch auf dem JFET-Source-Pad gestapelt (C) UnitedSiC) 


\begin{tabular}{|c|c|c|c|c|c|c|c|c|c|c|c|}
\hline \multirow[t]{2}{*}{ SiC-FET } & \multirow[t]{2}{*}{ Gehäuse } & \multirow{2}{*}{$\begin{array}{l}V_{\mathrm{DS}(\max )} \\
{[V]}\end{array}$} & \multirow{2}{*}{$\begin{array}{l}\text { ID }\left(100^{\circ} \mathrm{C}\right) \\
\text { [A] }\end{array}$} & \multirow{2}{*}{$\begin{array}{l}\mathbf{R}_{\mathrm{THJC}(\max )} \\
{[\mathrm{K} / \mathrm{W}]} \\
\end{array}$} & \multirow{2}{*}{$\begin{array}{l}\mathrm{R}_{\mathrm{DS}(O N)} \\
\left(25^{\circ} \mathrm{C}\right) \\
{[\mathrm{m} \Omega]}\end{array}$} & \multirow{2}{*}{$\begin{array}{l}\mathrm{R}_{\mathrm{DS}(\mathrm{ON})} \\
\left(125^{\circ} \mathrm{C}\right) \\
{[\mathrm{m} \Omega]}\end{array}$} & \multirow{2}{*}{$\begin{array}{l}\mathrm{R}_{\mathrm{DS}(\mathrm{ON})} \\
\left(175^{\circ} \mathrm{C}\right) \\
{[\mathrm{m} \Omega]}\end{array}$} & \multirow{2}{*}{$\begin{array}{l}C_{\text {oss }} \\
(800 \mathrm{~V}) \\
{[\mathrm{pF}]}\end{array}$} & \multirow{2}{*}{$\begin{array}{l}E_{\mathrm{ON}} \\
{[\mathrm{mJ}]}\end{array}$} & \multirow{2}{*}{$\begin{array}{l}E_{\text {ofF }} \\
{[\mathrm{mJ}]}\end{array}$} & \multirow{2}{*}{$\begin{array}{l}\text { Schalt- } \\
\text { zustände }\end{array}$} \\
\hline & & & & & & & & & & & \\
\hline UF3SC120009K4S & T0247-4L & 1200 & 120 & 0,19 & 8,6 & 13,3 & 18,2 & 395 & 3,5 & 0,7 & $\begin{array}{l}100 \mathrm{~A}, 800 \mathrm{~V}, \\
\mathrm{HB} 150 \mathrm{C}\end{array}$ \\
\hline UF3SC120016K4S & T0247-4L & 1200 & 77 & 0,29 & 16 & 24,8 & 33 & 243 & 2,82 & 0,15 & $\begin{array}{l}80 \mathrm{~A}, 800 \mathrm{~V}, \\
\mathrm{HB} 150 \mathrm{C}\end{array}$ \\
\hline UF3SC120016K3S & T0247-3L & 1200 & 77 & 0,29 & 16 & 24,8 & 33 & 243 & 3,35 & 0,67 & $\begin{array}{l}80 \mathrm{~A}, 800 \mathrm{~V}, \\
\mathrm{HB} 150 \mathrm{C}\end{array}$ \\
\hline UF3SC065007K4S & T0247-4L & 650 & 120 & 0,19 & 6,7 & 8,7 & 11 & 865 & 1,08 & 0,1 & $\begin{array}{l}80 \mathrm{~A}, 400 \mathrm{~V}, \\
\mathrm{HB} 150 \mathrm{C}\end{array}$ \\
\hline
\end{tabular}

TABELLE 1 Auswahl von SiC-FETs ( $\mathrm{R}_{\mathrm{DS}(\mathrm{ON})}$ : Drain-Source-Einschaltwiderstand; $\mathrm{C}_{\mathrm{OSs}}$ : Ausgangskapazität; $\mathrm{E}_{\mathrm{ON}}$, $\mathrm{E}_{\mathrm{OFF}}$ : Schaltenergie für An oder Aus; $\mathrm{V}_{\mathrm{DS}(\max )}$ : Maximale Drain-Source-Spannung; $\mathrm{I}_{\mathrm{D}}$ : Drain-Strom; $\mathrm{R}_{\mathrm{THJC( \operatorname {max } )}}$ : Maximale thermische Widerstandssperrschicht; HB: Halbleiterübergang) (@ UnitedSiC)

seine Gate-Spannung hoch ist. Hierdurch werden auch Gate und Source des JFETs kurzgeschlossen, sodass dieser zwangsweise eingeschaltet ist. Sinkt das Gate-Spannung des Mosfets auf Low, steigt dessen Drain-Spannung an, aber nur bis zu dem Punkt, an dem der JFET bei ungefähr $15 \mathrm{~V}$ abgeschaltet wird. Das Ergebnis ist ein selbstsperrender (normally-off) Baustein mit einfacher Ansteuerung des Gates. Darüber hinaus verfügt er über alle Vorteile eines SiCHalbleiters mit niedrigem Einschaltwiderstand, Hochspannungs- und Hochtemperaturbetrieb und einem integrierten Body-Diodeneffekt mit guten ReverseRecovery-Eigenschaften.

Kaskoden werden schon seit langer Zeit in eingesetzt. Ihre Entwicklung hat dazu geführt, dass auf JFETs basierende Kaskodenbausteine heute auch bei hohen Nennspannungen ausgesprochen niedrige Einschaltwiderstände erreichen. Damit kommen Kaskoden dem idealen Schalter ziemlich nahe. TABELLE 1 zeigt eine Auswahl von SiCFETs von UnitedSiC mit $\mathrm{R}_{\mathrm{DS}(\mathrm{ON})}$-Werten von gerade einmal 8,6 $\mathrm{m} \Omega$ für einen $1200-\mathrm{V}$ Baustein beziehungsweise $6,7 \mathrm{~m} \Omega$ für einen 650 -V-Baustein (beide bei $25{ }^{\circ} \mathrm{C}$ ). Alle Bausteine sind in TO-247-Gehäusen integriert; einige der Bausteine sind mit Vier-Leiter-Kelvin-Anschlüssen für eine optimale Gate-Ansteuerung ausgestattet.

\section{STEUERUNG INDUKTIVER LASTEN}

Systeme, die Halbleiterschalter zur Steuerung induktiver Lasten verwenden, wie zum Beispiel in Motorantrieben, müssen auch in der Lage sein, den Stromfluss umzukehren. In IGBT-Schaltungen ist hierfür normalerweise eine Hochspannungs-Paralleldiode erforderlich, um diesen Umkehrstromfluss zu ermöglichen. Dies verursacht Mehrkosten. Um einen effizienten Betrieb zu gewährleisten, müssen die Dioden auch eine hohe Leistung bieten und einen minimalen Reverse-Recovery-Energieverlust aufweisen. SiC-Mosfets enthalten eine integrierte Rückwärtsdiode. Damit können die zusätzlichen Kosten beim Hinzufügen einer Diode umgangen werden. Leider ist jedoch die Leistung durch einen hohen Vorwärtsspannungsabfall und erhebliche Rückspeiseverluste relativ schlecht.

Setzt man jedoch einen SiC-FET ein, so kann die Leistung verbessert werden. Er unterstützt den Umkehrstromfluss durch den Kanal ohne Reverse-RecoveryEffekte. Außerdem zeichnet er sich durch einen geringen Vorwärtsspannungsabfall über den bereits niedrigen Einschaltwiderstand aus. Der gestapelte Si-Mosfet im Gehäuse leitet ebenfalls in Rückwärtsrichtung, aber da es sich um einen optimierten Niederspannungstyp handelt, ist sein Body-Diodenabfall gering, und er trägt daher auch wenig zu den RecoveryVerlusten bei. Die neueste Generation von SiC-FETs eignet sich für die Steuerung von induktiven Lasten, mit geringeren Verlusten als beim herkömmlichen IGBTAnsatz und den zusätzlichen Vorteilen.

In TABELLE 2 sind die berechneten Verluste bei sechs Leistungsstufen im Vergleich zwischen einem IGBT-Modul nach dem aktuellen Stand der Technik und einem Paralleldiodenansatz mit verschiedenen SiC-FETs dargestellt. Wie TABELLE 2 zeigt, erreichen SiC-FETs bei dem typischen 50- bis 100-kW-Niveau eine fast vierfache Reduzierung der Verlustleistung und bei $200 \mathrm{~kW}$ eine fast dreifache Reduzierung. Bei Elektrofahrzeuganwendungen bedeutet dies, dass mehr Energie für die Traktions- motoren verfügbar ist, was wiederum eine größere Reichweite ermöglicht. Eine höhere Effizienz führt auch zu einem geringeren Kühlbedarf. Das führt zu kleineren Designs mit einfacheren und leichteren Kühlkörpern und damit zu einem geringeren Gesamtgewicht, was ebenfalls zu einer höheren Reichweite beiträgt. Die Verfügbarkeit von Bauelementen mit niedrigem Einschaltwiderstand in kostengünstigen, diskreten Gehäusen wird sowohl einen effizienteren als auch wirtschaftlicheren Ansatz für Inverter-Designs ermöglichen.

Dies gilt insbesondere mit dem Aufkommen von SiC-FET-Bauelementen der 4. Generation. Im Vergleich zu einem SiC-Mosfet kann ein SiC-FET der 4. Generation bei $25{ }^{\circ} \mathrm{C}$ eine um $50 \%$ bessere und bei $125^{\circ} \mathrm{C}$ eine um $40 \%$ bessere Kennzahl (Figure of Merit, FoM) erreichen. Dies ist besonders bei hart schaltenden Anwendungen von Bedeutung, bei denen die Ausgangskapazität $\mathrm{C}_{\text {oss }}$ schnell von einer hohen Spannung entladen wird, was zu einem hohen transienten Energieverlust führt.

SiC-FETs sind zudem widerstandsfähig gegen Kurzschlüsse. Der Kurzschlussstrom wird durch den JFET eingestellt und ist unempfindlich gegenüber den Parametern der gestapelten Mosfets. Durch Eigenerwärmung reduziert der positive Temperaturkoeffizient des JFETKanalwiderstands dann den Kurzschlussstrom weiter. Dadurch kann der Baustein längere Kurzschlusszeiten verkraften, bevor die Antriebselektronik den Kaskodenschalter sicher abschaltet. Durch diesen Effekt lassen sich SiC-FETs leicht zu einem automatischen Stromausgleich parallel schalten, was durch die relative Unempfindlichkeit der JFET-Schwellenspannung sowie der Reverse-Recovery- 


\section{ATZ live}

\section{Jetzt online anmelden!}

www.atzlive.de/aufa

Bei Rückfragen stehen wir Ihnen auch telefonisch zur Verfügung: Tel.: +49 611 7878-131

\section{Automatisiertes Fahren 2021}

Vom assistierten

zum autonomen Fahren

08. - 09. Juni 2021

Wiesbaden oder virtuell via Live-Stream

/ SENSE

Sensorik und Umfelderkennung

/ PLAN AND THINK

Situationen erkennen, analysieren, bewerten; Verhalten planen und entscheiden

I ACT

Funktionen des automatisierten

Fahrens umsetzen

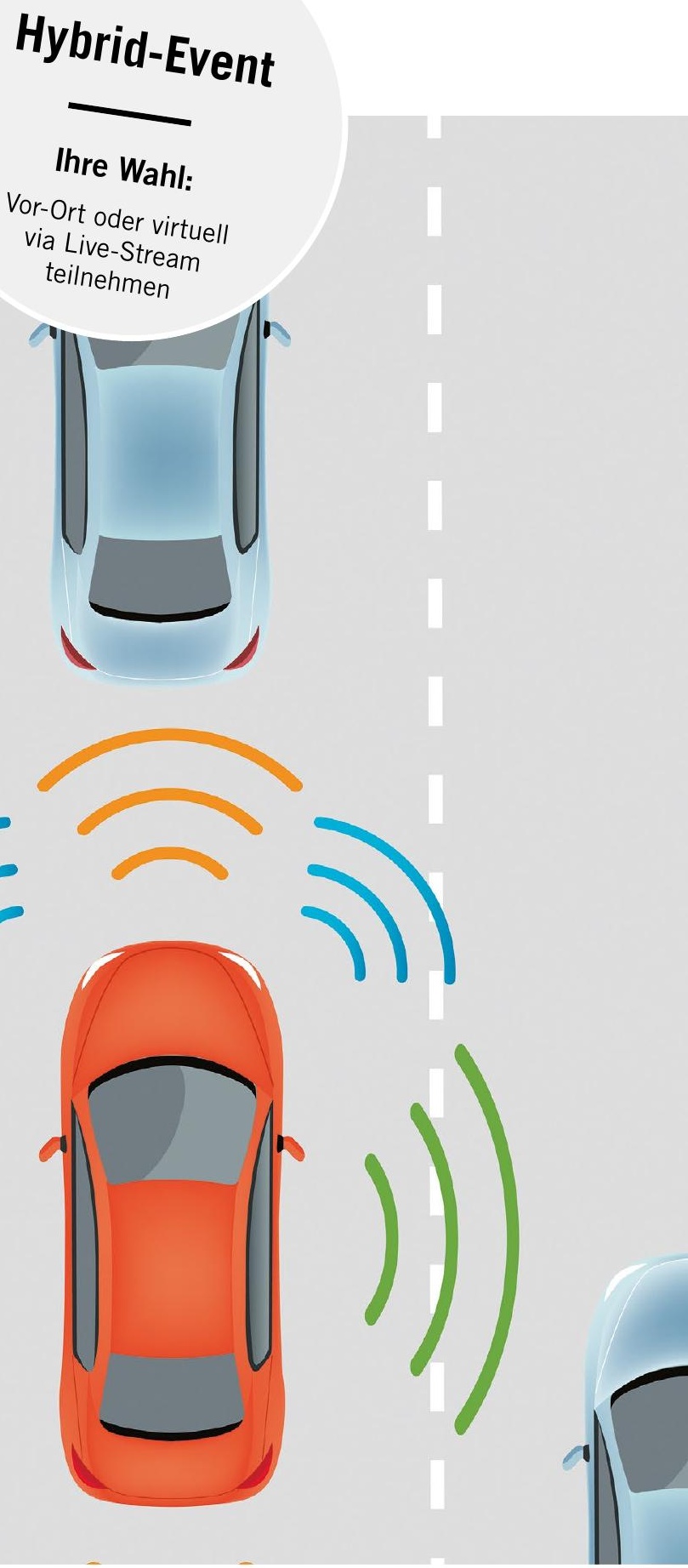




\begin{tabular}{|c|c|c|c|c|c|c|c|c|c|c|c|}
\hline \multirow{2}{*}{$\begin{array}{l}\text { Spannungs- } \\
\text { klasse }\end{array}$} & \multirow{2}{*}{$\begin{array}{l}\text { Komponen- } \\
\text { tentyp }\end{array}$} & \multirow[b]{2}{*}{ Chips/Schalter } & \multirow{2}{*}{$\begin{array}{l}\text { Bus- } \\
\text { span- } \\
\text { nung }\end{array}$} & \multirow{2}{*}{$\begin{array}{l}\text { Fre- } \\
\text { quenz }\end{array}$} & \multirow[b]{2}{*}{ Verlusttyp } & \multicolumn{6}{|c|}{ Ausgangsleistung } \\
\hline & & & & & & $50 \mathrm{~kW}$ & $100 \mathrm{~kW}$ & $150 \mathrm{~kW}$ & $200 \mathrm{~kW}$ & $250 \mathrm{~kW}$ & $300 \mathrm{~kW}$ \\
\hline \multirow{4}{*}{1200} & \multirow{4}{*}{ IGBT+Diode } & \multirow{4}{*}{ jeweils $100 \mathrm{~A} \times 4$} & \multirow{4}{*}{$800 \mathrm{~V}$} & \multirow{4}{*}{$8 \mathrm{kHz}$} & $\mathrm{P}_{\text {Zustand }}[\mathrm{W}]$ & 193 & 440 & 742 & 1097 & & \\
\hline & & & & & $\mathrm{P}_{\text {Schattend }}[\mathrm{W}]$ & 823 & 1191 & 1559 & 1927 & & \\
\hline & & & & & $\mathrm{P}_{\text {Gesamt }}[\mathrm{W}]$ & 1016 & 1613 & 2301 & 3024 & & \\
\hline & & & & & Wirkungsgrad [\%] & 97,97 & 98,37 & 98,47 & 98,49 & & \\
\hline \multirow{4}{*}{1200} & \multirow{4}{*}{ SiC-FET } & \multirow{4}{*}{ UF3SC120009K4S $\times 4$} & \multirow{4}{*}{$800 \mathrm{~V}$} & \multirow{4}{*}{$8 \mathrm{kHz}$} & $P_{\text {Zustand }}[W]$ & 67 & 270 & 608 & 1080 & & \\
\hline & & & & & $P_{\text {Schaltend }}[\mathrm{W}]$ & 185 & 218 & 261 & 313 & & \\
\hline & & & & & $\mathrm{P}_{\text {Gesamt }}[\mathrm{W}]$ & 252 & 488 & 869 & 1393 & & \\
\hline & & & & & Wirkungsgrad [\%] & 99,50 & 99,51 & 99,42 & 99,30 & & \\
\hline \multirow{4}{*}{1200} & \multirow{4}{*}{ SiC-FET } & \multirow{4}{*}{ UF3SC12009K4S × 6} & \multirow{4}{*}{$800 \mathrm{~V}$} & \multirow{4}{*}{$8 \mathrm{kHz}$} & $\mathrm{P}_{\text {Zustand }}[\mathrm{W}]$ & 45 & 180 & 405 & 720 & 1127 & 1621 \\
\hline & & & & & $\mathrm{P}_{\text {Schaltend }}[\mathrm{W}]$ & 265 & 293 & 327 & 368 & 415 & 469 \\
\hline & & & & & $\mathrm{P}_{\text {Geamt }}[\mathrm{W}]$ & 310 & 473 & 732 & 1088 & 1542 & 2090 \\
\hline & & & & & Wirkungsgrad [\%] & 99,38 & 99,53 & 99,51 & 99,46 & 99,23 & 98,96 \\
\hline \multirow{4}{*}{1200} & \multirow{4}{*}{ SiC-FET } & \multirow{4}{*}{ UF3SC120009K4S $\times 4$} & \multirow{4}{*}{$800 \mathrm{~V}$} & \multirow{4}{*}{$16 \mathrm{kHz}$} & $\mathrm{P}_{\text {Zustand }}[\mathrm{W}]$ & 67 & 270 & 608 & 1080 & & \\
\hline & & & & & $\mathrm{P}_{\text {Schaltend }}[\mathrm{W}]$ & 370 & 436 & 521 & 625 & & \\
\hline & & & & & $\mathrm{P}_{\text {Gesamt }}[\mathrm{W}]$ & 437 & 706 & 1129 & 1705 & & \\
\hline & & & & & Wirkungsgrad [\%] & 99,13 & 99,29 & 99,25 & 99,15 & & \\
\hline
\end{tabular}

TABELLE 2 Gesamtleitungs- $\left(P_{\text {Gesamt }}\right)$ und Schaltverluste $\left(P_{\text {Schaltend }}\right)$ beim Vergleich von IGBTs und SiC-FETs in Elektrofahrzeugen (@ UnitedSiC)

Charakteristik gegenüber Temperaturänderungen zusätzlich unterstützt wird.

\section{LADEN VON ELEKTROFAHRZEUGEN}

Die Ladeinfrastruktur ist ein weiteres entscheidendes Element bei der Einführung von Elektrofahrzeugen. Schnelles Laden erfordert eine hohe Leistung, die an das Fahrzeug geliefert und von diesem verwaltet werden muss. Das erfordert ein robustes On-Board-Ladegerät. SiC-FETs eignen sich für den Einsatz in Schnellladegeräten, wo sie sowohl in PFC-Frontends als auch in der DC/DC-Hauptwandlerstufe hohe Wirkungsgrade bieten.

SiC-Dioden werden bereits zur Realisierung der Ausgangsgleichrichtung in Hochspannungsladegeräten eingesetzt. Die Verwendung von SiC-FETS mit niedrigem Drain-Source-Einschaltwiderstand könnte sich jedoch als vorteilhafter erweisen. Beispielsweise wird eine SiCDiode bei einem Betriebsstrom von $100 \mathrm{~A}$ und einem Tastverhältnis von $50 \%$ Leitungsverluste von fast $100 \mathrm{~W}$ aufweisen, während der SiC-FET UF3SC065007K4S Leitungsverluste von nur $45 \mathrm{~W}$ erreicht. Darüber hinaus eröffnet die Synchrongleichrichtung die Möglichkeit eines bidirektionalen Stromflusses, sodass die Elektrofahrzeugbatterie beispielsweise für den Lastausgleich von Versorgungsunternehmen Strom ins Netz zurückführen kann, mit entsprechendem finanziellem Nutzen.

\section{RÜCKWÄRTSKOMPATIBILITÄT}

Da die SiC-FETs im drei- und vierpoligen TO-247-Gehäuse erhältlich sind, können sie einen Drop-in-Ersatz für viele IGBTs und Si-Mosfets in Motorantrieben darstellen. Dies führt zu einer hohen Effizienzsteigerung mit geringen Änderungen in der Schaltung, abgesehen vielleicht von Gate-Ansteuerwiderständen und kleinen Dämpfern (Snubbers) zur Anpassung der Schaltflanken. Die Anforderungen an die Gate-Ansteuerspannung sind unkritisch, typischerweise 0 bis $12 \mathrm{~V}$. Weitere Vorteile wie die Reduzierung vorhandener Dämpfungsglieder für geringere Verluste sowie das Weglassen von Paralleldioden in Designs, die ursprünglich auf IGBTs basierten, können in Betracht gezogen werden. Wie bei früheren SiC-FET-Generationen ermöglicht die hohe maximale Gate-Ansteuerspannung der Gen-4-Bausteine die Nachrüstung von Bauteilen in bestehende Schaltungen, um IGBTs, Si-Mosfets und SiC-Mosfets ohne oder mit nur geringen Änderungen an der Anordnung der Gate-Ansteuerung auszutauschen und so eine sofortige Leistungssteigerung zu erzielen.

\section{ZUSAMMENFASSUNG}

Der Absatz von Elektrofahrzeugen steigt stetig. Ein großes Manko ist derzeit aber noch die Reichweite der Fahrzeuge bei rein elektrischem Betrieb. Um die Verluste bei der Übertragung der elektrischen Energie zu minimieren und damit die Reichweite der Fahrzeuge zu steigern, hat United Silicon Carbide Siliziumkarbidhalbleiter entwickelt, mit denen effizientere elektrische Antriebsstränge und Leistungsbauteile realisiert werden können.

\section{LITERATURHINWEISE}

[1] iea: Global EV Outlook 2020. Technology report - June 2020. Online: https://www.iea.org/ reports/global-ev-outlook-2020, aufgerufen: 1. Februar 2021

[2] UnitedSiC: SiC FETs. Online: https://unitedsic. com/group/sic-fets/, aufgerufen: 1. Februar 2021 


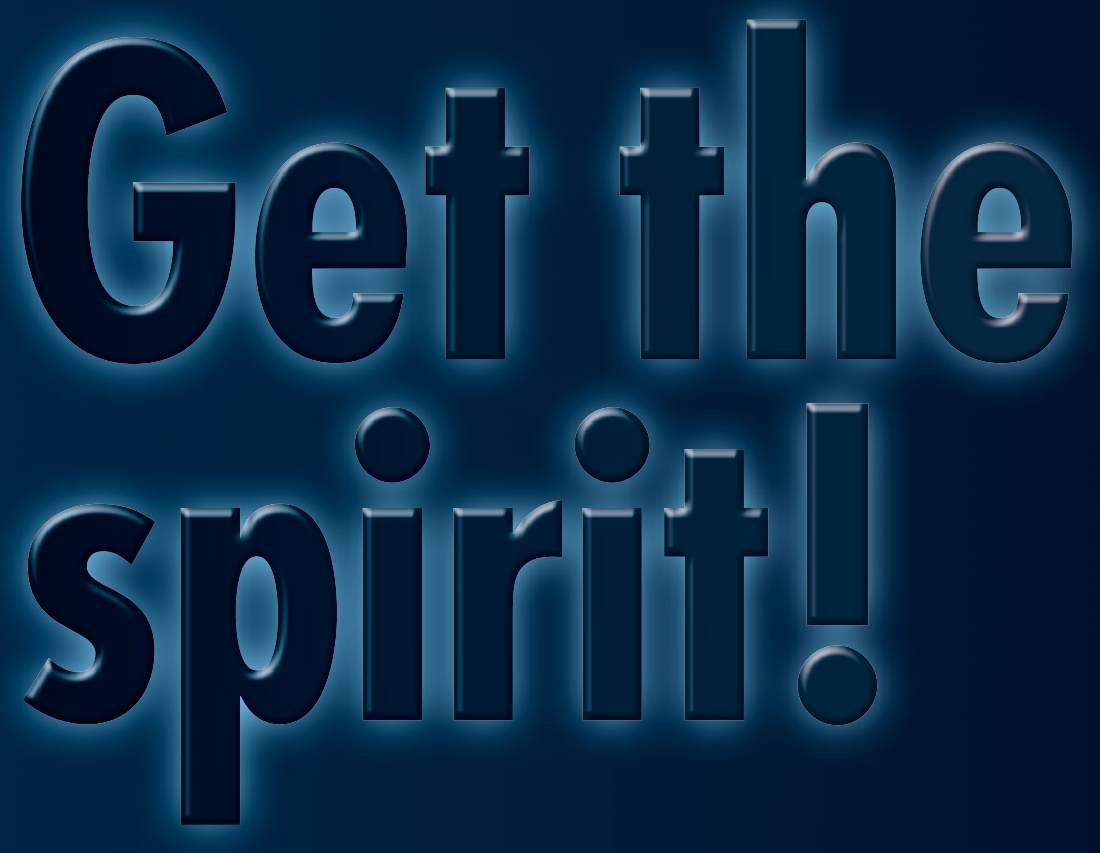

\section{Starten Sie durch und sichern Sie sich die Basis für lhren Erfolg.}

Das neue Magazin Sales Excellence ist die führende Plattform für Vertriebsexperten und bietet innovative Techniken für erfolgreiche Spitzenreiter, die die Chancen und Herausforderungen im Vertrieb der Gegenwart und der Zukunft im Blick haben. Nutzen Sie die gesamten Potenziale von Print, E-Magazin, Social Media und der digitalen Wissensdatenbank von Springer Professional.

\section{Jetzł 2 Ausgaben kostenlos testen.}

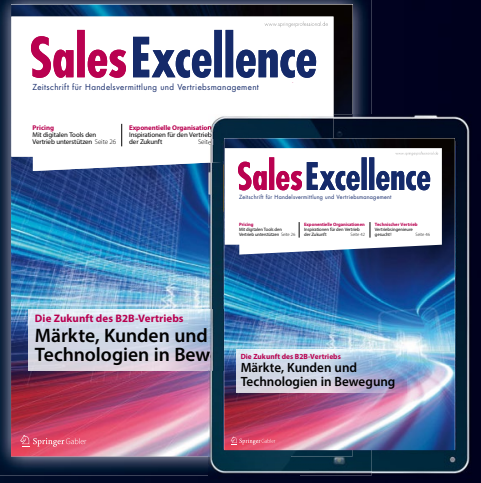

Für Vertriebsingenieure der Automobilbranche. 